\title{
Detection of ischemia with early myocardial perfusion imaging: You see more if you watch before
}

\author{
Riccardo Liga, $M D{ }^{a}$ and Alessia Gimelli, $M D^{b}$ \\ ${ }^{a}$ Cardio-thoracic and Vascular Department, University Hospital of Pisa, Pisa, Italy \\ ${ }^{\mathrm{b}}$ Fondazione Toscana, Gabriele Monasterio, Pisa, Italy
}

Received Mar 7, 2016; accepted Mar 7, 2016

doi:10.1007/s12350-016-0470-y

\section{See related article, pp. 1149-1156}

Current medical guidelines for the management of subjects with stable angina make the evaluation of the presence and extent of inducible myocardial ischemia as a mandatory step in the clinical workflow. ${ }^{1}$ Moreover, the precise quantification of the myocardial ischemic burden is widely believed as a necessary action also in the management of patients with ascertained coronary artery disease (CAD). ${ }^{2}$ As a matter of fact, in the last decades solid evidence has demonstrated that patients with limited myocardial ischemia can be safely treated conservatively (i.e., with medical therapy alone), ${ }^{3}$ while symptomatic subjects with relevant inducible ischemia should be managed more aggressively and submitted, if indicated, to coronary revascularization. ${ }^{4,5}$

The evaluation of the presence of myocardial ischemia can be performed either non-invasively, mostly by means of provocative tests, or invasively, such as with the assessment of fractional flow reserve. ${ }^{5}$ In this scenario, myocardial perfusion scintigraphy (MPS) by means of single-photon emission computed tomography (SPECT) allows a combined assessment of regional myocardial blood flow distribution coupled with left ventricular (LV) functional parameters in a completely non-invasive manner, ${ }^{3,6}{ }^{8}$ offering the chance of unmasking the presence of significant CAD with a relevant accuracy. ${ }^{9,10}$ Accordingly, recent reports have consistently demonstrated that the integrated evaluation

Reprint requests: Alessia Gimelli, MD, Fondazione Toscana Gabriele Monasterio, Via Moruzzi 1, 56124 Pisa, Italy; gimelli@ftgm.it J Nucl Cardiol 2017;24:1157-60.

1071-3581/\$34.00

Copyright (C) 2016 American Society of Nuclear Cardiology. of myocardial perfusion and LV systolic and diastolic functional measures may further increase the accuracy of SPECT imaging in detecting CAD, possibly allowing a more accurate risk stratification of patients with suspected or known disease. ${ }^{11,12}$

However, despite these obvious clinical advantages, MPS is characterized by two major technical drawbacks, such as the cumulative radiation exposure ${ }^{13}$ and the lengthiness of the imaging protocol, ${ }^{14}$ that classically limit its diffusion and make the clinician frequently favor other non-invasive imaging modalities.

Recent advance in SPECT technology have effectively addressed these major aspects of nuclear imaging, offering the chance of further increasing its implementation. Above all, the introduction of dedicated cardiac cameras, designed with cadmium-zinc-telluride (CZT) detectors, probably represents one of the most relevant novelties in the nuclear field. ${ }^{15}$

In fact, due to their increased photon sensitivity and spatial resolution if compared to traditional SPECT cameras, CZT devices allow obtaining an excellent image quality despite a consistently reduced radiotracer's injected dose. ${ }^{16}$ Accordingly, different reports have clearly demonstrated that the introduction of CZT devices may allow to perform a stress myocardial perfusion scan with a radiation dose close to $1 \mathrm{mSv}$ and a low-dose stress/rest myocardial imaging protocol with a cumulative dose of roughly 3 to $4 \mathrm{mSv} .{ }^{16,17}$ Moreover, the favorable technical specifics of CZT devices have also allowed a consistent reduction of image acquisition times, offering the chance to perform an excellently tolerated, high-quality, test lasting 4 to 6 minutes. ${ }^{16,18,19}$

Despite the astonishing reduction of the effective acquisition times, the overall duration of a CZT imaging protocol, as in the case of a traditional SPECT scan, is mainly determined by the waiting intervals between the injection of the radiopharmaceutical and the acquisition itself. $^{15,16}$ In fact, those time periods between the 
acquisitions are necessary to allow the complete disappearance of the radiotracer from both the blood pool and the sub-diaphragmatic organs (i.e., liver, spleen, and bowel), reducing the incidence of most of the classical artifacts of myocardial nuclear imaging. ${ }^{20}$

Reports on the possibility of consistently reducing those waiting intervals, that may be as long as 45 to 60 minutes before each acquisition, and to increase the overall speediness of SPECT imaging are discordant, leaving relevant indecision on the subject. ${ }^{6,21}$ However, an earlier acquisition of MPS images could allow a better detection not only of perfusion abnormalities, but of more precocious, subtle, alterations of LV function (i.e., alterations of myocardial contractile and/or diastolic functions) that might help to better stratify the severity and extension of risk area. ${ }^{6}$

The actual relevance of this topic in the era of new CZT devices was specifically addressed by Meyer and colleagues and is reported in the present number of the Journal of Nuclear Cardiology. ${ }^{22}$

In this study, patients submitted to either exercise (30 subjects), vasodilator (20 subjects) or resting (20 subjects) MPS acquisitions underwent both an early scan, performed 3 to 5 minutes after the radiotracer's injection, and a delayed acquisition, performed after the standard 45 minutes after the administration of ${ }^{99 \mathrm{~m}} \mathrm{Tc}$ Sestamibi. The scans were performed on a semi-upright position with a dedicated D-SPECT cardiac camera, equipped with 9 collimated, pixilated cadmium-zinctelluride (CZT) detector columns, mounted vertically in $90^{\circ}$ geometry. The obtained pairs of images were compared visually for overall image quality and the presence and extent of extracardiac activity (recorded on a subjective scale). Finally, the presence and location of regional myocardial ischemia was compared in early and delayed CZT scans and the presence of visual disagreement between the two acquisition was determined.

Accordingly, no significant difference in overall image quality and severity of extracardiac activity was revealed between early and delayed scans, with the majority of CZT acquisitions graded as good to excellent. Moreover, despite the contained time interval between the radiopharmaceutical's injection and the acquisition, the great majority of the CZT scans (83\%) didn't show any extracardiac activity.

Interestingly, when the presence and location of myocardial ischemia was considered, a consistent divergence between early and delayed CZT acquisitions was found, particularly in the case of exercise (37\% of the cases) and vasodilator (60\%) stress tests. Despite this finding, the prevalence of inferior LV wall artifacts on early images (i.e., perfusion defects absent on delayed acquisitions), as indirect measures of relatively increased sub-diaphragmatic activity, was minimal (only
2 cases) and the overall inter-observer agreement between early and delayed scans was highly acceptable $(87 \%)$. The authors concluded that, while early and delayed CZT acquisitions were generally comparable with respect of image quality, the presence of a perfusion defect on early acquisitions might sometimes have an artifactual nature that would request a second, delayed, scan.

In this respect, despite the authors of the study have to be commended for having addressed such a clinically relevant topic, a number of limitations reduce the generalizability of its results.

First of all, the small number of the patients studied and the absence of an accepted gold standard, such as invasive coronary angiography (ICA), towards which MPS results could be validated represent relevant drawbacks of the study.

As a matter of fact, by definition, an imaging artifact represents a perfusion defect that takes place in the absence of a coronary stenosis and can be explained by the occurrence of coexisting extracardiac conditions (i.e., sub-diaphragmatic activity). In the study by Meyer et al., the absence of a diagnostic gold standard limits the assumption by which any perfusion defect that is present in only one CZT acquisition, either early or delayed, must invariably represent an imaging artifact.

In this respect, it has been previously shown, using conventional SPECT cameras and a similar radiotracer $\left({ }^{99 \mathrm{~m}} \mathrm{Tc}-\right.$ Tetrofosmin), that perfusion data obtained early after the radiotracer's administration might present an increased sensitivity and overall accuracy in unmasking the presence of significant coronary stenosis on ICA. ${ }^{6}$

However, until now, data on the feasibility of early MPS acquisitions, also performed with a CZT-based dedicated cardiac device, have been inconclusive, showing, in some cases the occurrence of significant perfusion artifacts when the acquisition was started earlier than 5 minutes after the administration of the radiotracer. ${ }^{21,23}$

Nevertheless, as previously mentioned, early MPS imaging might offer the chance to perform not only a better evaluation of severity and extension of perfusion abnormalities, but a more physiological assessment of LV functional status under stress conditions. ${ }^{711}$ In fact, it has been classically shown that myocardial ischemia results in reversible alterations of LV diastolic, at first, and contractile functions, subsequently.

In this respect, MPS imaging offers to obtain accurate and reproducible measures of both LV systolic and diastolic functions, allowing to better risk stratify patients with inducible myocardial ischemia and to integrate the assessment of regional myocardial perfusion with more clinically oriented functional markers (i.e., ejection fraction and peak filling rate). ${ }^{11,12}$ 
Unfortunately, those functional measures of myocardial ischemia are classically transient and may disappear before the traditional, "delayed", acquisition. Accordingly, an early CZT scan has the obvious advantage to allow a more precocious evaluation of those subtle alterations of cardiac function, such as ischemia-related transitory systolic or diastolic dysfunction, that may unmask the presence of significant coronary lesions. In line with these evidences, recent reports have suggested how the development of poststress LV diastolic impairment (i.e., a reduced peak filling rate), as evaluated by early CZT imaging, may unmask the presence of coronary atherosclerosis, additively to the presence of myocardial perfusion abnormalities. ${ }^{12,24}$

In conclusion, despite the absence of a gold standard such as invasive coronary angiography and the lack of the assessment of LV functional parameters, the proof-of-concept study by Meyer et al. shades light on the possibility to consistently reduce the overall MPS acquisition time without effecting image quality.

Further, more powered investigations, are eagerly awaited to definitively establish whether early image acquisitions through CZT devices will only represent a logistic benefit for the patient or also allow a significant increase in the diagnostic accuracy of SPECT evaluations.

\section{Disclosures}

Alessia Gimelli and Riccardo Liga have no conflicts of interest to disclose.

\section{References}

1. Members Task Force, Montalescot G, Sechtem U, Achenbach S, Andreotti F, Arden C, Budaj A, et al. ESC guidelines on the management of stable coronary artery disease: The Task Force on the management of stable coronary artery disease of the European Society of Cardiology. Eur Heart J 2013;2013(34):2949-3003.

2. Authors/Task Force members, Windecker S, Kolh P, Alfonso F, Collet JP, Cremer J, Falk V, et al. 2014 ESC/EACTS Guidelines on myocardial revascularization: The Task Force on Myocardial Revascularization of the European Society of Cardiology (ESC) and the European Association for Cardio-Thoracic Surgery (EACTS)Developed with the special contribution of the European Association of Percutaneous Cardiovascular Interventions (EAPCI). Eur Heart J 2014;35:2541-619.

3. Hachamovitch R, Hayes SW, Friedman JD, Cohen I, Berman DS. Comparison of the short-term survival benefit associated with revascularization compared with medical therapy in patients with no prior coronary artery disease undergoing stress myocardial perfusion single photon emission computed tomography. Circulation 2003;107:2900-7.

4. Shaw LJ, Berman DS, Maron DJ, Mancini GB, Hayes SW, Hartigan PM, et al. Optimal medical therapy with or without percutaneous coronary intervention to reduce ischemic burden: Results from the Clinical Outcomes Utilizing Revascularization and Aggressive Drug Evaluation (COURAGE) trial nuclear substudy. Circulation 2008;117:1283-91.

5. De Bruyne B, Fearon WF, Pijls NH, Barbato E, Tonino P, Piroth $\mathrm{Z}$, et al. Fractional flow reserve-guided PCI for stable coronary artery disease. N Engl J Med 2014;371:1208-17.

6. Giorgetti A, Rossi M, Stanislao M, Valle G, Bertolaccini P, Maneschi A, et al. Feasibility and diagnostic accuracy of a gated SPECT early-imaging protocol: A multicenter study of the Myoview Imaging Optimization Group. J Nucl Med 2007;48:1670-5.

7. Gimelli A, Liga R, Pasanisi EM, Giorgetti A, Marras G, Favilli B, et al. Evaluation of left ventricular diastolic function with a dedicated cadmium-zinc-telluride cardiac camera: Comparison with Doppler echocardiography. Eur Heart J Cardiovasc Imaging 2014;15:972-9.

8. Giorgetti A, Masci PG, Marras G, Rustamova YK, Gimelli A, Genovesi D, et al. Gated SPECT evaluation of left ventricular function using a CZT camera and a fast low-dose clinical protocol: Comparison to cardiac magnetic resonance imaging. Eur J Nucl Med Mol Imaging 2013;40:1869-75.

9. Kapur A, Latus KA, Davies G, Dhawan RT, Eastick S, Jarritt PH, et al. A comparison of three radionuclide myocardial perfusion tracers in clinical practice: The ROBUST study. Eur J Nucl Med Mol Imaging 2002;29:1608-16.

10. Gimelli A, Liga R, Duce V, Kusch A, Clemente A, Marzullo P. Accuracy of myocardial perfusion imaging in detecting multivessel coronary artery disease: A cardiac CZT study. J Nucl Cardiol 2016;. doi:10.1007/s12350-015-0360-8.

11. Gimelli A, Liga R, Giorgetti A, Kusch A, Pasanisi EM, Marzullo P. Relationships between myocardial perfusion abnormalities and poststress left ventricular functional impairment on cadmium-zinctelluride imaging. Eur J Nucl Med Mol Imaging 2015;42:994-1003.

12. Gimelli A, Liga R, Bottai M, Pasanisi EM, Giorgetti A, Fucci S, et al. Diastolic dysfunction assessed by ultra-fast cadmium-zinctelluride cardiac imaging: Impact on the evaluation of ischaemia. Eur Heart J Cardiovasc Imaging 2015;16:68-73.

13. Lindner O, Pascual TN, Mercuri M, Acampa W, Burchert W, Flotats A, et al. Nuclear cardiology practice and associated radiation doses in Europe: Results of the IAEA Nuclear Cardiology Protocols Study (INCAPS) for the 27 European countries. Eur J Nucl Med Mol Imaging 2016;43:718-28.

14. Henzlova MJ, Duvall WL, Einstein AJ, Travin MI, Verberne HJ. ASNC imaging guidelines for SPECT nuclear cardiology procedures: Stress, protocols, and tracers. J Nucl Cardiol 2016. doi: 10.1007/s12350-015-0387-x.

15. Buechel RR, Herzog BA, Husmann L, Burger IA, Pazhenkottil $\mathrm{AP}$, Treyer $\mathrm{V}$, et al. Ultrafast nuclear myocardial perfusion imaging on a new gamma camera with semiconductor detector technique: First clinical validation. Eur J Nucl Med Mol Imaging 2010;37:773-8.

16. Gimelli A, Bottai M, Giorgetti A, Genovesi D, Kusch A, Ripoli A, et al. Comparison between ultrafast and standard single-photon emission CT in patients with coronary artery disease: A pilot study. Circ Cardiovasc Imaging 2011;4:51-8.

17. Einstein AJ, Blankstein R, Andrews H, Fish M, Padgett R, Hayes SW, et al. Comparison of image quality, myocardial perfusion, and left ventricular function between standard imaging and single-injection ultra-low-dose imaging using a high-efficiency SPECT camera: The MILLISIEVERT study. J Nucl Med 2014;55:1430-7.

18. Herzog BA, Buechel RR, Katz R, Brueckner M, Husmann L, Burger IA, et al. Nuclear myocardial perfusion imaging with a cadmium-zinc-telluride detector technique: Optimized protocol for scan time reduction. J Nucl Med 2010;51:46-51. 
19. Ben-Haim S, Almukhailed O, Neill J, Slomka P, Allie R, Shiti D, et al. Clinical value of supine and upright myocardial perfusion imaging in obese patients using the D-SPECT camera. J Nucl Cardiol 2014;21:478-85.

20. Jain D, Wackers FJ, Mattera J, McMahon M, Sinusas AJ, Zaret BL. Biokinetics of technetium- 99 m-tetrofosmin: Myocardial perfusion imaging agent: implications for a one-day imaging protocol. J Nucl Med 1993;34:1254-9.

21. Askew JW, Miller TD, Ruter RL, Jordan LG, Hodge DO, Gibbons RJ, et al. Early image acquisition using a solid-state cardiac camera for fast myocardial perfusion imaging. J Nucl Cardiol 2011;18:840-6.

22. Meyer C, Weinmann P. Validation of early image acquisitions following Tc-99m sestamibi injection using a semiconductors camera of cadmium-zinc-telluride. J Nucl Cardiol 2016. doi: 10.1007/s12350-016-0499-y.

23. Berman DS, Kang X, Tamarappoo B, Wolak A, Hayes SW, Nakazato R, et al. Stress thallium-201/rest technetium-99m sequential dual isotope high-speed myocardial perfusion imaging. JACC Cardiovasc Imaging 2009;2:273-82.

24. Gimelli A, Liga R, Pasanisi EM, Casagranda M, Marzullo P. Myocardial ischemia in the absence of obstructive coronary lesion: The role of post-stress diastolic dysfunction in detecting early coronary atherosclerosis. J Nucl Cardiol 2016. doi: 10.1007/s12350-016-0456-9. 